\title{
Movement Correction Method for Human Brain PET Images: Application to Quantitative Analysis of Dynamic ${ }^{18}$ F-FDDNP Scans
}

\author{
Mirwais Wardak ${ }^{1,2}$, Koon-Pong Wong ${ }^{1}$, Weber Shao ${ }^{1}$, Magnus Dahlbom ${ }^{1}$, Vladimir Kepe ${ }^{1}$, Nagichettiar Satyamurthy ${ }^{1}$, \\ Gary W. Small ${ }^{3-6}$, Jorge R. Barrio ${ }^{1}$, and Sung-Cheng Huang ${ }^{1,2}$ \\ ${ }^{I}$ Department of Molecular and Medical Pharmacology, David Geffen School of Medicine at UCLA, Los Angeles, California; \\ ${ }^{2}$ Department of Biomathematics, David Geffen School of Medicine at UCLA, Los Angeles, California; ${ }^{3}$ Department of Psychiatry \\ and Behavioral Sciences, David Geffen School of Medicine at UCLA, Los Angeles, California; ${ }^{4}$ Semel Institute for Neuroscience and \\ Human Behavior, David Geffen School of Medicine at UCLA, Los Angeles, California; ${ }^{5}$ Mary S. Easton Center for Alzheimer's \\ Disease Research, Los Angeles, California; and ${ }^{6} U C L A$ Center on Aging, Los Angeles, California
}

\begin{abstract}
Head movement during a PET scan (especially a dynamic scan) can affect both the qualitative and the quantitative aspects of an image, making it difficult to accurately interpret the results. The primary objective of this study was to develop a retrospective image-based movement correction (MC) method and evaluate its implementation on dynamic 2(1-\{6-[(2-18F-fluoroethyl)(methyl)amino]-2-naphthyl $\}$ ethylidene)malononitrile $\left({ }^{18} \mathrm{~F}-\mathrm{FDDNP}\right)$ PET images of cognitively intact controls and patients with Alzheimer's disease (AD). Methods: Dynamic ${ }^{18} \mathrm{~F}$-FDDNP PET images, used for in vivo imaging of $\beta$-amyloid plaques and neurofibrillary tangles, were obtained from $12 \mathrm{AD}$ patients and 9 age-matched controls. For each study, a transmission scan was first acquired for attenuation correction. An accurate retrospective $\mathrm{MC}$ method that corrected for transmission-emission and emission-emission misalignments was applied to all studies. No restriction was assumed for zero movement between the transmission scan and the first emission scan. Logan analysis, with the cerebellum as the reference region, was used to estimate various regional distribution volume ratio (DVR) values in the brain before and after MC. Discriminant analysis was used to build a predictive model for group membership, using data with and without MC. Results: MC improved the image quality and quantitative values in ${ }^{18} \mathrm{~F}-\mathrm{FDDNP} P E T$ images. In this subject population, no significant difference in DVR value was observed in the medial temporal (MTL) region of controls and patients with $A D$ before MC. However, after MC, significant differences in DVR values in the frontal, parietal, posterior cingulate, MTL, lateral temporal $(\mathrm{LTL})$, and global regions were seen between the 2 groups $(P<0.05)$. In controls and patients with $A D$, the variability of regional DVR values (as measured by the coefficient of variation) decreased on average by more than $18 \%$ after MC. Mean DVR separation between controls and patients
\end{abstract}

Received Mar. 4, 2009; revision accepted Nov. 2, 2009.

For correspondence or reprints contact: Mirwais Wardak, Department of Molecular and Medical Pharmacology, David Geffen School of Medicine at UCLA, B2-085E CHS, 10833 Le Conte Ave., Los Angeles, CA 90095-6948.

E-mail: mwardak@mednet.ucla.edu

Guest Editor: Adriaan A. Lammertsma, VU University Medical Center COPYRIGHT $\odot 2010$ by the Society of Nuclear Medicine, Inc. with AD was higher in frontal, MTL, LTL, and global regions after MC. Group classification by discriminant analysis based on ${ }^{18} \mathrm{~F}-$ FDDNP DVR values was markedly improved after MC. Conclusion: The streamlined and easy-to-use MC method presented in this work significantly improves the image quality and the measured tracer kinetics of ${ }^{18} \mathrm{~F}-\mathrm{FDDNP}$ PET images. The proposed MC method has the potential to be applied to PET studies on patients having other disorders (e.g., Down syndrome and Parkinson's disease) and to brain PET scans with other molecular imaging probes.

Key Words: head movement correction; ${ }^{18} \mathrm{~F}-\mathrm{FDDNP}$; Alzheimer's disease; discriminant analysis; distribution volume ratio

J Nucl Med 2010; 51:210-218

DOI: 10.2967/jnumed.109.063701

H ead movement in PET is an issue that clinicians and research scientists have to address if they want to accurately quantify the pharmacokinetic analysis of metabolic information contained in a PET image. This is particularly true in the elderly, with increased significance in patients with dementia or movement disorders. The development of methods to correct for head movement in PET data is also especially important as the spatial resolution of PET cameras continues to improve, and movement artifacts become more evident (1).

Typical dynamic PET scans with molecular imaging probes usually last for at least an hour, a lengthy procedure for elderly patients but one that is necessary for accurate brain pharmacokinetics. Head movement during the scan can not only introduce a loss in spatial resolution in the image but may also lead to a loss of information and even useless data (2). Part of the problem is movement-induced misalignment between transmission and emission scans as the subject moves from his or her initial position at the start 
of the study, which is when the transmission scan is acquired. This movement will cause an incorrect attenuation correction factor (ACF) matrix to be applied to each uncorrected emission frame of the dynamic image. The integrity of the reconstructed PET data will hence be compromised (3).

Moreover, when dynamic images are acquired, movement within an emission frame and movement between emission frames can cause additional complications. Classical compartmental model analysis, along with its linearized or graphical counterparts (4), may give erroneous values for physiologic parameters (e.g., distribution volume ratio [DVR] or metabolic flux) derived from such methods, because movement between frames can alter the validity of image-derived blood and tissue time-activity curves. The motivation to address such a continuing problem in PET is thus greatly merited.

One way to reduce head movement during a PET scan is to use a stereotactic head restraint or thermoplastic mask. However, these devices are not able to completely eliminate head movement (5) and could produce significant discomfort or claustrophobia in elderly patients, which would compromise the studies. Therefore, a better solution is needed.

The primary purpose of this work was to develop a retrospective image-based head movement correction (MC) method and then evaluate its validity on $2-\left(1-\left\{6-\left[\left(2-{ }^{18} \mathrm{~F}-\right.\right.\right.\right.$ fluoroethyl)(methyl)amino]-2-naphthyl $\}$ ethylidene)malononitrile ( ${ }^{18}$ F-FDDNP) PET images of cognitively intact controls and patients with Alzheimer's disease (AD). ${ }^{18} \mathrm{~F}$-FDDNP is a hydrophobic molecular probe used for in vivo imaging of $\beta$-amyloid plaques and neurofibrillary tangles $(6,7)$, the neuropathologic hallmarks of $\mathrm{AD}$. The MC method would have to not only correct for transmission-emission misalignment but also correct for emission-emission misalignment if present in these PET studies. In addition, the MC method has to be easy to use to make it practical for common use. The effects of head movement on tissue timeactivity curves and parametric DVR images would also have to be addressed.

\section{MATERIALS AND METHODS}

\section{Clinical Assessments}

The retrospective MC method was evaluated and validated on $12 \mathrm{AD}$ patients and 9 controls. Table 1 summarizes the clinical characteristics in this subject population. All subjects underwent comprehensive neurologic and psychiatric evaluations as previously reported (6). The Mini-Mental State Examination (MMSE) (8) was used as one of the clinical tools for detecting cognitive impairment and assessing its severity. Subjects with AD met the standard diagnostic criteria for AD (9). Control subjects had normal cognitive functioning for their age and did not meet the diagnostic criteria for AD. In accordance with the procedures of the Human Subjects Protection Committee at UCLA, written informed consent was obtained from all subjects or their legal representatives.

\begin{tabular}{|c|c|c|}
\hline Parameter & Control & $A D$ \\
\hline$n$ & 9 & 12 \\
\hline Sex (male/female) & $6 / 3$ & $6 / 6$ \\
\hline Age (y) & $69 \pm 8(62-85)$ & $74 \pm 8(61-85)$ \\
\hline MMSE $^{\star}$ & $29.4 \pm 0.7(28-30)$ & $17.8 \pm 6.8(4-25)$ \\
\hline $\begin{array}{l}\text { Injected } \\
\text { dose (MBq) }\end{array}$ & $\begin{array}{l}383.3 \pm 24.2 \\
\quad(351.5-418.1)\end{array}$ & $\begin{array}{l}382.6 \pm 29.9 \\
\quad(318.2-421.8)\end{array}$ \\
\hline \multicolumn{3}{|c|}{$\begin{array}{l}{ }^{*} \text { Scores range from } 0 \text { to } 30 \text {, with higher scores indicating } \\
\text { better cognitive functioning. } \\
\text { Values are mean } \pm S D \text {, with range in parentheses. }\end{array}$} \\
\hline
\end{tabular}

18F-FDDNP PET Protocol

Dynamic ${ }^{18} \mathrm{~F}-\mathrm{FDDNP}$ PET scans were acquired with an ECAT EXACT HR + scanner (Siemens/CTI). A curved head-holder made of carbon fiber was attached to the patient bed. The intrinsic spatial resolution of the scanner was $4.5 \mathrm{~mm}$ in full width at half maximum in the center of the field of view. Using a set of external rotating ${ }^{68} \mathrm{Ge}$ rod sources, we first acquired a transmission scan for $20 \mathrm{~min}$ in 2-dimensional mode to correct for photon attenuation occurring along each line of response in the emission scan. ${ }^{18} \mathrm{~F}$ FDDNP (382.95 $\pm 27.07 \mathrm{MBq})$, as synthesized by methods previously reported (10), was injected intravenously as a bolus, and a dynamic emission scan $(6 \times 30 \mathrm{~s}, 4 \times 3 \mathrm{~min}, 5 \times 10 \mathrm{~min}$, and $3 \times 20 \mathrm{~min}$ ) was initiated. Dynamic images were reconstructed using filtered backprojection with a Hann filter at a cutoff frequency of $0.3 \times$ Nyquist and a zoom factor of 3.5. The final reconstructed volume had a spatial resolution of $9 \mathrm{~mm}$ in full width at half maximum and a matrix size of $128 \times 128$ that consisted of 63 planes, resulting in a voxel size of $1.47 \times 1.47 \times$ $2.43 \mathrm{~mm}$. The same reconstruction parameters were used in the MC method.

\section{Procedure}

The head MC method consists of 2 major parts (Fig. 1). The first part properly corrects for the attenuation in each of the emission frames of the dynamic image. The second part then aligns each of the properly attenuation-corrected (AC) emission frames to a common reference frame. The MC method involves stepping back and forth between projection space and image space, because the rigid-body alignment routine in the freely available SPM2 software (11) must be done in image space. The reslicing of image volumes was also done in SPM2 using trilinear interpolation. The Clinical Applications Programming Package (CAPP; Siemens) was used to reconstruct images. The full procedure, which has been streamlined and made easy to use, consists of the following steps:

- The first part of the MC method starts by segmenting out the head-holder from the transmission image because the headholder stayed stationary when the patient's head moved. This is done by manually drawing a contour around the headholder in ImageJ (12) and then masking it out. This is the only user-dependent task within the MC method, although an easy one.

- The original transmission image without the head-holder ( TX $_{\text {ORIGINAL }}$ ) is then coregistered (as a rigid body) to 


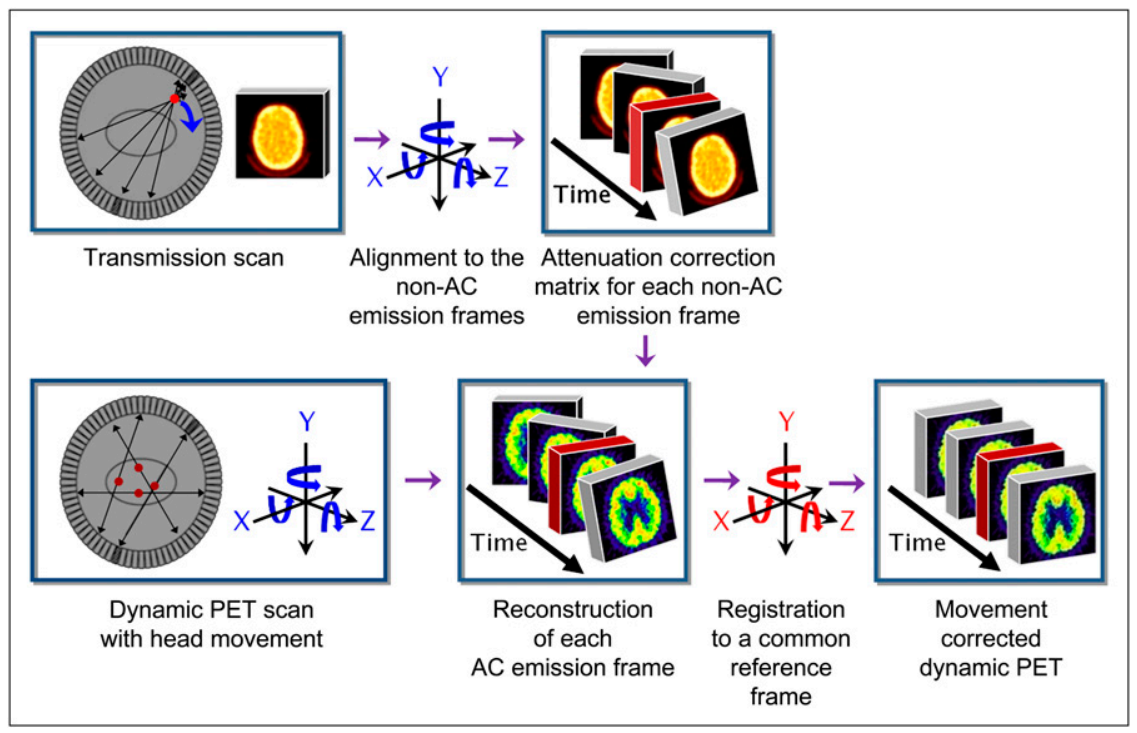

FIGURE 1. General illustrated overview of retrospective MC method proposed in this study. time-activity curve derived from the cerebellar cortex as the reference input for time points between 15 and $125 \mathrm{~min}$. The slope of the linear portion of the Logan plot is the DVR, which is equal to the distribution volume of the tracer in an ROI divided by the distribution volume of the tracer in the reference region (4).

ROIs were separately drawn before and after MC on the early summed ${ }^{18} \mathrm{~F}$-FDDNP image (frames $1-7$ ), which resembled tracer perfusion (14), and then applied to the DVR image to calculate various regional DVR values in the brain. The volume of the ROIs ranged from 0.2 to $2.0 \mathrm{~cm}^{3}$. Image visualization and ROI analyses were performed using CAPP software. Regions included in the analysis were (left and right) frontal, parietal, posterior cingulate, medial temporal (MTL), lateral temporal (LTL), subcortical white matter, and cerebellum. Furthermore, each regional DVR value was expressed as a volume-weighted average of the left and right regions, and global DVR values were then calculated as straight averages for all these regional values (6), excluding the cerebellum and subcortical white matter. The effects of head movement on tracer time-activity curves were also assessed.

\section{Statistical Analysis}

All statistical analyses were performed using SPSS, version 17.0 (SPSS Inc.) for Windows (Microsoft). The results are reported as the mean \pm SD (unless otherwise noted) and are regarded as statistically significant if $P$ was less than 0.05 .

Group Comparisons. Group differences in regional DVR values between controls and patients with $\mathrm{AD}$ were tested with the Mann-Whitney test (2-tailed). A nonparametric test was chosen because of our small group size and because this type of test makes fewer assumptions about the sample data characteristics (i.e., samples do not have to be drawn from normally distributed populations with equal variance).

Discriminant Analysis. Discriminant analysis was used to build a predictive model for group membership (15). Given a set of independent variables, discriminant analysis attempts to find linear combinations of those variables that best separate the groups of subjects. These combinations are called discriminant functions and have the form displayed in the following equation:
To quantify ${ }^{18}$ F-FDDNP binding, Logan graphical analysis (4) was applied to generate DVR parametric images, using the 


$$
\mathrm{D}=\mathrm{b}_{1} \mathrm{x}_{1}+\mathrm{b}_{2} \mathrm{x}_{2}+\ldots+\mathrm{b}_{\mathrm{n}} \mathrm{x}_{\mathrm{n}}+\mathrm{C}
$$

where $\mathrm{D}$ is the discriminant score formed by the discriminant function, $b_{n}$ are the discriminant coefficients, $x_{n}$ are the predictor variables, and $\mathrm{C}$ is a constant.

Moreover, the discriminant function is generated from a sample of cases for which group membership is known and can then be applied to new cases that have measurements for the predictor variables but have unknown group membership (15). To assess the predictive performance of the discriminant function, a classification table was generated summarizing the percentage and number of subjects classified correctly and incorrectly. Although the classifications based on the cases used to create the model may be looked upon as too optimistic, in the sense that their classification rate is inflated, a cross-validated section of the table attempts to correct this by classifying each subject while leaving it out of the model calculations (15). Cross-validation thus gives an approximately unbiased estimate of the true error rate, although a relatively noisy one (16).

\section{RESULTS}

\section{Group Characteristics}

The groups were shown to be statistically matched for age $(P>0.05)$. The mean MMSE scores for the control and $\mathrm{AD}$ groups were $29.4 \pm 0.7$ and $17.8 \pm 6.8$, respectively $(P<0.001)$.

\section{Image Processing and Analysis}

Figure 2 illustrates how head movement during a dynamic brain PET can cause misalignment between the transmission and the emission scans. The first part of the MC procedure described in this article corrects for this problem. Qualitative differences in ${ }^{18} \mathrm{~F}-\mathrm{FDDNP}$ DVR images before and after $\mathrm{MC}$ are shown in Figure 3. The DVR image of an AD patient with considerable head movement (e.g., AD patient 2 in Table 2) before MC was subject to image artifacts (i.e., increased scalp uptake and asymmetric ${ }^{18} \mathrm{~F}$-FDDNP binding near the top of the head as seen in the coronal view) because of transmission-emission misalignment. Scalp uptake of ${ }^{18}$ F-FDDNP, in general, is patient-specific, but it should not be abnormally high and asymmetric as seen in the DVR image before MC. However, after the MC procedure was applied, the image quality of the DVR image was improved, and ${ }^{18}$ F-FDDNP signal was more clearly defined in frontal, striatal, thalamic, and temporal regions. For a control subject with minor head movement (e.g., Control subject 1 in Table 2), the MC procedure did not introduce any artifacts or image degradation to the DVR image. As a result, the image integrity of the original DVR image was maintained.

Transformation parameters ( 3 translations, 3 rotations) for Control subject 1 and $\mathrm{AD}$ patient 2 are shown in Supplemental Figure 1 (supplemental materials are available online only at http://jnm.snmjournals.org). A lefthanded coordinate system (11) was used to describe the transformation parameters. The severity of the head movement for all subjects in this study was determined by not

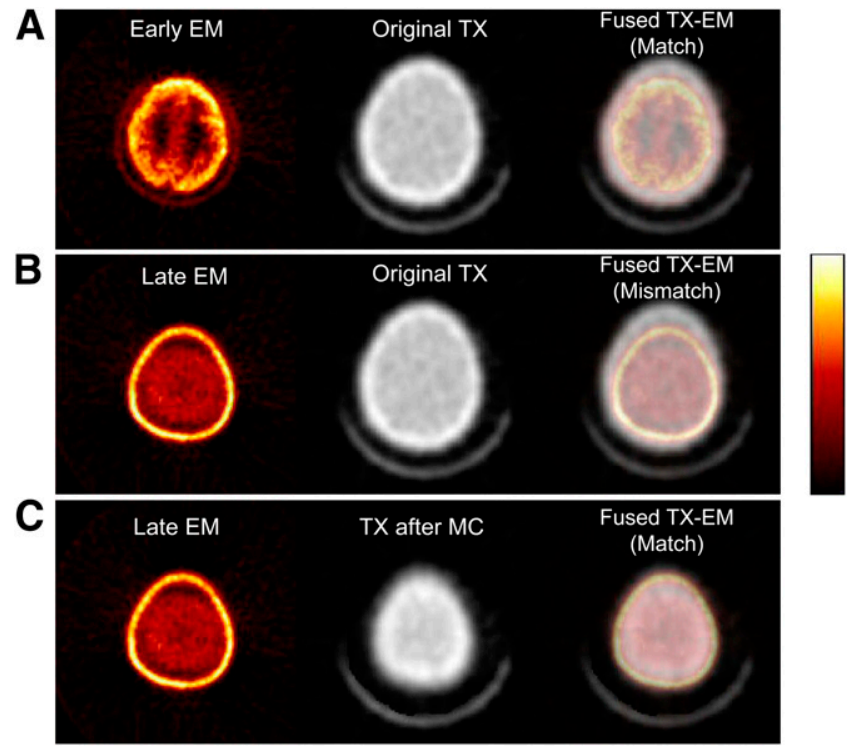

FIGURE 2. Head movement can cause transmissionemission misalignment during dynamic brain PET. (A) Early non-AC emission frame is shown to be fused well with transmission image taken at start of study. Both images are shown at same transaxial plane. (B) Late non-AC emission frame is now fused with original transmission image at same transaxial plane as before. Because subject's head moves away from PET gantry after acquisition of early non-AC emission frame, mismatched transmission-emission would cause an incorrect ACF matrix to be applied to late non-AC emission frame. (C) After first part of MC method has been applied, the original transmission image is matched to the late emission image, producing an accurate ACF matrix that can be used to properly reconstruct the late emission frame.

only examining the transformation parameters but also looking at the fused images of $\mathrm{TX}_{\text {ORIGINAL }}$, compared with each of the non-AC emission frames. The primary translational direction affected by head movement for both controls and patients with AD was the longitudinal $z$-axis (i.e., the patient's superior-inferior axis).

Displacement maps in Figure 4 show the individual displacement of each voxel in the reference non-AC emission frame as it is coregistered to a late non-AC emission frame. The displacement is defined as:

$$
\text { Displacement }=\sqrt{\operatorname{trans}_{\mathrm{x}}^{2}+\operatorname{trans}_{\mathrm{y}}^{2}+\operatorname{trans}_{\mathrm{z}}^{2}} \quad \text { Eq. } 2
$$

where $\operatorname{trans}_{\mathrm{x}}, \operatorname{trans}_{\mathrm{y}}$, and $\operatorname{trans}_{\mathrm{z}}$ are the absolute translations undergone by a voxel in each directional axis (i.e., a patient's left-right, anterior-posterior, and superiorinferior axes, respectively) during the registration process. For Control subject 1 (Fig. 4A), the displacements were small $(<4 \mathrm{~mm})$. As a result, regional time-activity curves and Logan plots for this subject showed no noticeable changes before and after MC (Supplemental Figs. 2A-2D). For AD patient 2 (Fig. 4B), the displacements were as large as $24 \mathrm{~mm}$, with increasing regional displacements occur- 


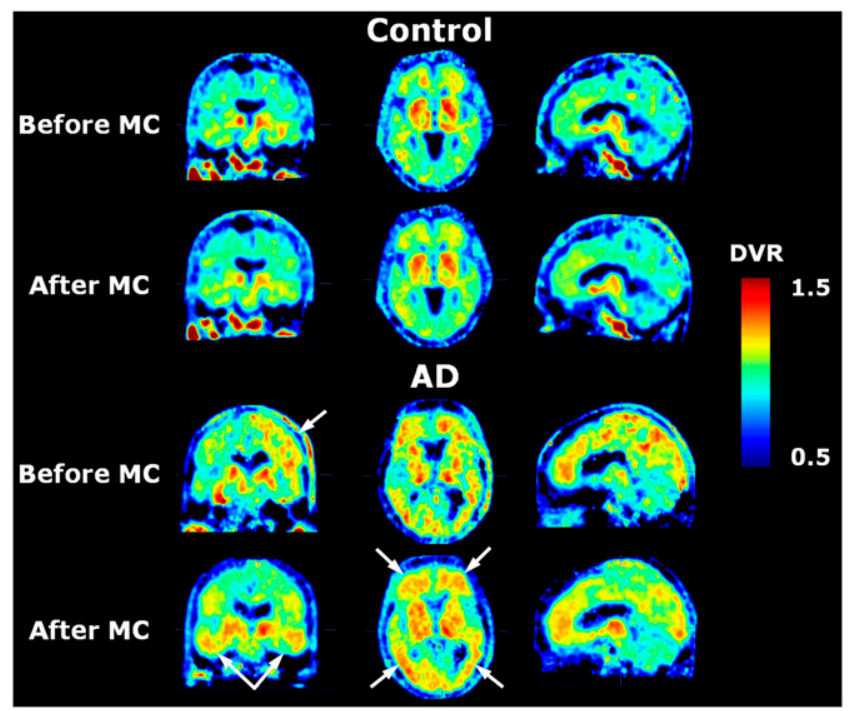

FIGURE 3. For a control subject with negligible head movement, MC method did not introduce any apparent image degradation to ${ }^{18} \mathrm{~F}-\mathrm{FDDNP}$ DVR image. Before MC, DVR image of an AD patient with considerable head movement was subject to image artifacts (i.e., abnormally high scalp uptake and asymmetric ${ }^{18}$ F-FDDNP binding near top of head as seen in coronal view) due to transmissionemission misalignment. However, after MC, image quality of DVR image was improved. All images correspond approximately to same cross-sectional locations in their respective coronal, transaxial, and sagittal views.

ring in the following order: (cerebellum, parietal, and posterior cingulate) $(\sim 12-13 \mathrm{~mm})<$ (striatum, thalamus, MTL, and LTL) $(\sim 14-15 \mathrm{~mm})<$ (frontal) $(\sim 17-18 \mathrm{~mm})$. As a result, regional time-activity curves before $\mathrm{MC}$ were distorted for this subject, and regional DVR values from the Logan plots differed before and after MC (Supplemental Figs. 2E-2H). The displacement pattern for each subject in each group was dependent on the subject's individual head movements during the scan and might not necessarily mirror what is shown in Figure 4.

The mean displacement (calculated from all the voxels in the head) of the reference non-AC emission frame as it is coregistered to each of the remaining frames in the dynamic image is plotted in Figure 5 for Control subject 1 and AD patient 2. The maximum of the mean displacement between the reference frame and any non-AC emission frame is shown in Table 2 for all subjects. The maximum of the maximum displacement (undergone by a single voxel in the head) between the reference frame and any non-AC emission frame is also shown in Table 2 for all subjects.

\section{Group Comparisons}

The results of the group comparisons in regional DVR values before and after MC are summarized in Table 3 and plotted in Supplemental Figure 3. In this subject population, significant differences between controls and patients with $\mathrm{AD}$ were found in frontal, parietal, posterior cingulate,
TABLE 2. Displacement During Dynamic ${ }^{18} \mathrm{~F}-\mathrm{FDDNP}$ PET Studies in Control Subjects and AD Patients

Displacement ( $\mathrm{mm})$

\begin{tabular}{ccc} 
Group & Maximum of mean* & Maximum of maximum \\
\cline { 2 - 3 } Control subjects & \\
1 & 2.10 (frame 18) & 3.53 (frame 18) \\
2 & 2.32 (frame 18) & 3.73 (frame 4) \\
3 & 12.42 (frame 17) & 18.10 (frame 17) \\
4 & 4.13 (frame 18) & 8.90 (frame 17) \\
5 & 2.27 (frame 17) & 5.01 (frame 5) \\
6 & 4.62 (frame 15) & 9.45 (frame 15) \\
7 & 2.60 (frame 18) & 5.68 (frame 5) \\
8 & 2.20 (frame 5) & 4.98 (frame 5) \\
9 & 2.45 (frame 4) & 5.99 (frame 4) \\
$\mathrm{AD}$ patients & & \\
1 & 6.29 (frame 3) & 10.80 (frame 3) \\
2 & 15.30 (frame 18) & 23.59 (frame 18) \\
3 & 15.89 (frame 18) & 30.58 (frame 18) \\
4 & 4.73 (frame 13) & 10.97 (frame 15) \\
5 & 2.66 (frame 15) & 4.54 (frame 15) \\
6 & 2.60 (frame 17) & 3.70 (frame 3) \\
7 & 8.68 (frame 18) & 15.73 (frame 18) \\
8 & 3.66 (frame 18) & 8.03 (frame 18) \\
9 & 4.60 (frame 18) & 6.63 (frame 18) \\
10 & 2.98 (frame 18) & 7.09 (frame 18) \\
11 & 2.44 (frame 18) & 5.42 (frame 17) \\
12 & 15.11 (frame 18) & 20.18 (frame 18)
\end{tabular}

*Maximum of mean displacement (for which mean is taken over all voxels in brain) between reference frame and any non$\mathrm{AC}$ emission frame.

${ }^{\dagger}$ Maximum of maximum displacement (undergone by single voxel in brain) between reference frame and any non-AC emission frame.

Non-AC emission frames for which maximum occurs are shown in parentheses.

LTL, and global regions before MC $(P<0.05)$. MTL and subcortical white matter regions did not show significant differences before MC. By contrast, all regions showed significant differences between control subjects and $\mathrm{AD}$ patients after MC $(P<0.05)$, except for subcortical white matter. Additionally, the $P$ values from the Mann-Whitney test for frontal, parietal, posterior cingulate, MTL, LTL, and global DVR values decreased after MC, with the MTL region having the most prominent decrease (a decrease of more than $90 \%$ in $P$ value).

In control subjects, the variability of DVR values (as measured by the coefficient of variation [CV]) in frontal, parietal, posterior cingulate, MTL, LTL, subcortical white matter, and global regions decreased on average by $18 \%$ after MC. In subjects with AD, the variability of DVR values in frontal, MTL, LTL, and subcortical white matter regions decreased on average by $36 \%$ after MC. The decrease in the $\mathrm{CV}$ for subcortical white matter was especially apparent in the $\mathrm{AD}$ group, with a decrease of more than $70 \%$ from its original value. The DVR separation between controls and patients with $\mathrm{AD}$ (as measured by the separation in the 
A
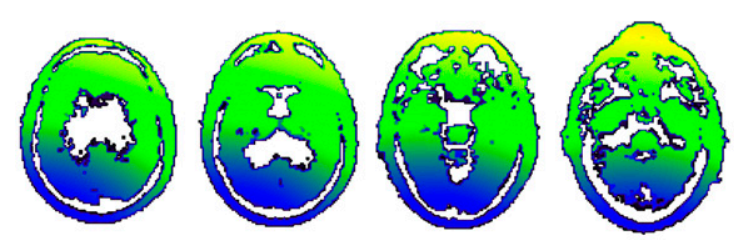
( $\mathrm{mm}$ )

B
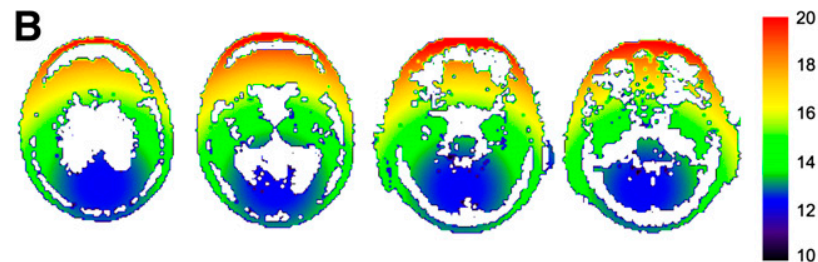

FIGURE 4. Displacement maps for control subject with minor head movement $(A)$ and $A D$ patient with significant head movement (B). Each map shows displacement undergone by each voxel in reference non-AC emission frame as it is coregistered to last non-AC emission frame in dynamic image. Note difference in scale for both color bars.

means of the 2 groups) was higher in frontal, MTL, LTL, and global regions after $\mathrm{MC}$ than before $\mathrm{MC}$.

\section{Discriminant Analysis}

Supplemental Figure 4 shows the scatter plots of the discriminant scores (before and after MC) for the control and $\mathrm{AD}$ groups using frontal, parietal, posterior cingulate, MTL, and LTL collectively as predictor regions in the discriminant function. Note the complete separation between the 2 groups after MC, which leads to $100 \%$ overall accuracy in the classification performance by the discriminant function when cross-validation is not applied.

Table 4 summarizes the overall accuracy, sensitivity, and specificity in group classification performance (with and without cross-validation) for various combinations of predictor regions before and after MC. The overall accuracy in addition to the sensitivity and specificity improved after MC.

\section{DISCUSSION}

A retrospective image-based head MC method that corrected for transmission-emission and emission-emission misalignments was proposed and investigated in this study. To make it practical for common use, the MC method had to be easy to use and not add tremendous burden to any human brain PET study. The shape of a human head changes little with movement, so rigid-body transformations were used to model the relative positions during a dynamic PET scan (11). Two images were matched by finding the translations and rotations that optimized some matching function of the images (11). This type of MC method is of value to those who want to extract the fine details of tracer behavior in a PET image but are restricted in doing so because of the degradation present in

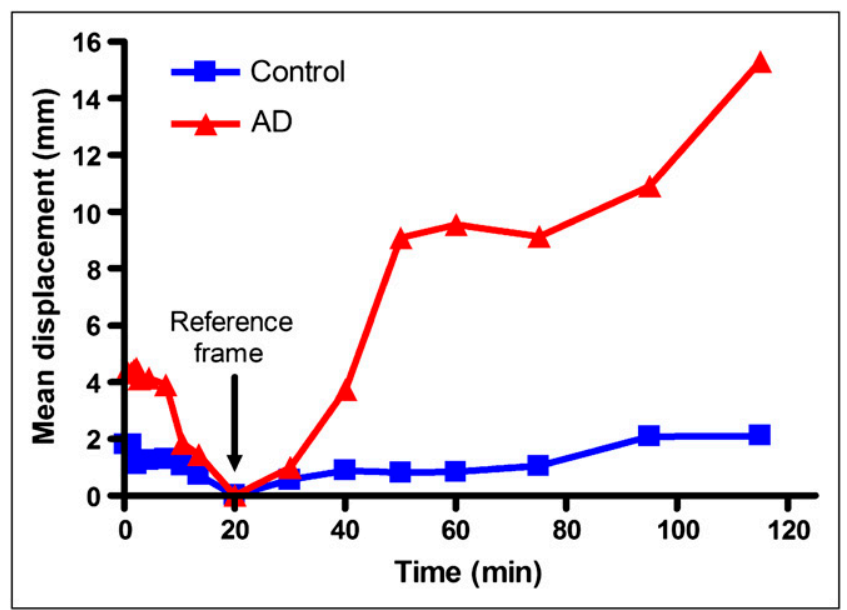

FIGURE 5. Mean displacement of reference non-AC emission frame as it is coregistered to each remaining frame of the dynamic image as shown for a control subject with negligible head movement and an $A D$ patient with large head movement. Mean was calculated from displacements of all voxels in head.

the image caused by patient movement. With the proposed MC method presented in this article, valuable data once hidden in a brain PET image can come to light, making otherwise questionable studies useful.

Various strategies have been used to address the problems of patient head movement in PET $(1,2,17-22)$. An attractive method of late has been the acquisition of PET data in list-mode while simultaneously tracking the patient's head movement with an optical motion-tracking system $(1,22)$. The optical motion-tracking system emits infrared light and detects the translational and rotational information of the head during image acquisition from the light that is reflected back from markers positioned on the patient's head. Each detected event in the list-mode data is then corrected for by the movement information provided by the motion-tracking system, and the image is reconstructed thereafter (1). A big advantage of this method is its tracer independence (i.e., there is no reliance on the PET data for determining the head movement). However, there are technical issues with this method that still need to be addressed (1). Also, optical-tracking systems are of no help in trying to retrospectively correct for head movement in PET images previously acquired. For these cases, imagebased methods would offer a more practical solution.

One of the limitations of image-based methods (besides their susceptibility to the quality of the PET data) is that they do not account for motion within a frame. However, this problem can be minimized by shortening the frame duration in the PET protocol. It must be cautioned, though, that excessive shortening of the frame duration might also increase the noise due to lower counting statistics. Furthermore, in the MC method presented in this article, there is no assumption or restriction set on having no movement 
TABLE 3. Logan DVR Values and CVs for Various Regions in Control and AD Groups Before and After MC

\begin{tabular}{|c|c|c|c|c|c|c|c|c|c|c|}
\hline \multirow[b]{3}{*}{ Region } & \multicolumn{5}{|c|}{ Before MC } & \multicolumn{5}{|c|}{ After MC } \\
\hline & \multicolumn{2}{|c|}{ Control } & \multicolumn{2}{|c|}{$A D$} & \multirow[b]{2}{*}{$P^{+}$} & \multicolumn{2}{|c|}{ Control } & \multicolumn{2}{|c|}{$A D$} & \multirow[b]{2}{*}{$P^{\dagger}$} \\
\hline & Mean DVR & $\% \mathrm{CV}^{*}$ & Mean DVR & $\% \mathrm{CV}^{*}$ & & Mean DVR & $\% \mathrm{CV}^{*}$ & Mean DVR & $\% \mathrm{CV}^{\star}$ & \\
\hline FRT & 1.032 & 4.55 & 1.148 & 5.11 & 0.0003 & 1.029 & 3.69 & 1.153 & 3.83 & 0.0001 \\
\hline PAR & 1.078 & 3.90 & 1.173 & 3.13 & 0.0016 & 1.072 & 3.17 & 1.161 & 3.60 & 0.0006 \\
\hline PCG & 1.112 & 5.03 & 1.212 & 3.65 & 0.0012 & 1.088 & 4.18 & 1.185 & 4.04 & 0.0007 \\
\hline MTL & 1.151 & 5.80 & 1.218 & 5.63 & 0.0817 & 1.127 & 4.44 & 1.207 & 4.83 & 0.0077 \\
\hline LTL & 1.100 & 5.60 & 1.177 & 4.42 & 0.0142 & 1.088 & 4.60 & 1.172 & 2.86 & 0.0012 \\
\hline SWM & 1.004 & 3.73 & 0.989 & 11.13 & 0.6441 & 1.010 & 3.64 & 1.008 & 3.25 & 0.9151 \\
\hline Global & 1.095 & 4.19 & 1.186 & 2.57 & 0.0010 & 1.082 & 3.07 & 1.176 & 2.97 & 0.0002 \\
\hline
\end{tabular}

between the transmission scan and the first emission scan, as is required by some other methods $(18,19,21)$. This restriction was removed by the alignment of the transmission image to the non-AC emission frames using the mutual information maximization criterion. Although mutual information is a powerful algorithm for the registration of multimodality images (and that worked well in our study), the optimization of other cost functions may be more appropriate for other tracers for which there is too little "mutual information" between the transmission image and the reference emission frame or between the emission frames themselves.

Recently, Costes et al. (20) investigated a coregistrationbased frame-realignment method for dynamic PET images using simulated ${ }^{11} \mathrm{C}$-raclopride PET data. Upon optimizing the choice of target volume and similarity criterion, a correction strategy was designed that took into account transmission-emission misalignment and the realignment of the individual time frames. Their optimal method consisted of using non-AC emission images and the cross-correlation criterion.

Moreover, Mourik et al. (21) evaluated 4 different offline frame-by-frame MC methods. Their optimal method (based on simulated ${ }^{11} \mathrm{C}$-flumazenil and $(R)-{ }^{11} \mathrm{C}$-PK11195 studies) consisted of aligning non-AC emission frames to a summed image of the early non-AC frames $(0-3 \mathrm{~min})$. They then used a common attenuation map to reconstruct a series of aligned AC emission images. The study assumed there was no patient motion during the early emission frames or between the transmission scan and the start of the emission scan. Although both of these studies mirrored our study in certain respects, there were some important differences: our method was derived from real patient data, not simulated data; our method specifically accounted for the attenuation due to the head-holder; normalized mutual information was the matching criterion used in our study; our method did not necessarily assume no mismatch between the transmission scan and the early frames of the emission scan; and we worked with ${ }^{18} \mathrm{~F}$ instead of ${ }^{11} \mathrm{C}$-radiolabeled probes.

With regard to the results reported in this paper, we saw that in Supplemental Figure 1 the head movements of Control subject 1 were relatively negligible, whereas the head movements of AD patient 2 could not be ignored, especially along the longitudinal $z$-axis. The DVR images of Control subject 1 before and after MC were similar (Fig. 3 ) and showed no apparent signs of image degradation that might have been introduced by the MC procedure. This should be the case if the MC procedure worked as it should,

TABLE 4. Overall Accuracy, Sensitivity, and Specificity in Classification Performance Before and After MC Using Various Combinations of Predictor Regions

\begin{tabular}{|c|c|c|c|c|c|c|}
\hline \multirow[b]{2}{*}{ Predictor region } & \multicolumn{3}{|c|}{ Before MC } & \multicolumn{3}{|c|}{ After MC } \\
\hline & $\begin{array}{c}\text { Overall } \\
\text { accuracy (\%) }\end{array}$ & Sensitivity (\%) & Specificity (\%) & $\begin{array}{c}\text { Overall } \\
\text { accuracy (\%) }\end{array}$ & Sensitivity (\%) & Specificity (\%) \\
\hline $\mathrm{FRT}+\mathrm{PAR}+\mathrm{PCG}+\mathrm{MTL}+\mathrm{LTL}$ & $95(91)$ & $92(92)$ & $100(89)$ & $100(95)$ & $100(92)$ & $100(100)$ \\
\hline $\mathrm{FRT}+\mathrm{MTL}+\mathrm{PAR}$ & $95(86)$ & $100(92)$ & 89 (78) & $100(95)$ & $100(92)$ & $100(100)$ \\
\hline $\mathrm{FRT}+\mathrm{MTL}+\mathrm{PCG}$ & $95(91)$ & $100(100)$ & 89 (78) & $100(95)$ & $100(92)$ & $100(100)$ \\
\hline FRT + PAR + PCG & $91(91)$ & $92(92)$ & $89(89)$ & $100(95)$ & $100(92)$ & $100(100)$ \\
\hline $\mathrm{FRT}+\mathrm{MTL}+\mathrm{LTL}$ & $91(91)$ & $92(92)$ & $89(89)$ & $100(95)$ & $100(92)$ & $100(100)$ \\
\hline $\mathrm{MTL}+\mathrm{LTL}$ & $76(71)$ & $83(75)$ & $67(67)$ & $86(86)$ & $92(92)$ & $78(78)$ \\
\hline
\end{tabular}


because the subject had relatively little head movement to begin with. However, this does not mean that all control subjects will have negligible head movement. For example, there were control subjects in this study whose head movements were substantial and could not be ignored (e.g., Control subject 3 in Table 2). The MC procedure should thus be applied to all subjects in a brain PET study, regardless of whether head movements were apparent during the scan.

Previous PET studies have shown significantly higher ${ }^{18}$ F-FDDNP binding in the frontal, parietal, and temporal regions of the brain in patients with $\mathrm{AD}$ than in older control subjects without cognitive impairment (6). After $\mathrm{MC}$, the image quality of the ${ }^{18} \mathrm{~F}$-FDDNP DVR images in subjects who moved was improved, and ${ }^{18}$ F-FDDNP binding in the aforementioned regions was more clearly defined. Quantitatively, this can be seen in the increased separation of the mean DVR values between controls and patients with AD in frontal, MTL, LTL, and global regions (Table 3; Supplemental Fig. 3). This increased separation explained in part why the discriminant analysis performed better after MC. Another reason for the improved performance was the considerable decrease (ranging from $42 \%$ to $91 \%$ ) in $P$ values after MC for frontal, parietal, posterior cingulate, MTL, LTL, and global regions. These 2 reasons allowed for the calculation of a more refined discriminant function that was used to correctly classify individuals from the sampled population. The resulting discriminant function can thus be of significant help to diagnose new AD cases based on ${ }^{18} \mathrm{~F}$-FDDNP DVR images.

Additionally, before MC, we saw that the MTL and subcortical white matter regions did not show significant differences between control subjects and AD patients. Because the MTL lobe is the brain region affected the earliest in AD (23), these results can be interpreted on the basis of the possibility that elderly control subjects may already have significant pathology present, even in the absence of neuropsychiatric symptoms. However, and even though it is possible that control subjects may have elevated MTL lobe signal with ${ }^{18}$ F-FDDNP PET, we saw that after MC the MTL region did show a significant difference $(P=0.0077)$ between control subjects and AD patients whereas differences in subcortical white matter remained insignificant between the 2 groups. The MTL region is thus sensitive to head movement. The fact that DVR values in subcortical white matter did not differ significantly between the 2 groups also offers the possibility of using subcortical white matter as a reference region for ${ }^{18} \mathrm{~F}-\mathrm{FDDNP}$ Logan analysis.

Moreover, the variability in regional DVR values decreased after MC. This decrease was most apparent in subcortical white matter in the AD group. MC should be considered if subcortical white matter is used as a reference region for Logan analysis, because its DVR is highly variable without $\mathrm{MC}$ in $\mathrm{AD}$. The reduction in within-group $\mathrm{CV}$ would affect the design considerations of an experiment and the costs involved, because the sample size needed for detecting a given percentage change between means is a function of the CV (16). In addition, regional timeactivity curves were distorted in subjects with large head movement when compared with the kinetic data after MC. This is a problem because the Logan plots of distorted time-activity curves would yield less reliable DVR values. The proposed MC method would thus be a significant contributor to the precision of the data.

\section{CONCLUSION}

The retrospective image-based MC method described in this article significantly improves the image quality and the measured tracer kinetics of ${ }^{18}$ F-FDDNP PET images. Reliable DVR estimations and increased mean DVR separation in frontal, MTL, LTL, and global regions between control subjects and AD patients are the results of using such a method. The refined discriminant functions derived from movement-corrected data add value to clinicians and researchers who want to use ${ }^{18}$ F-FDDNP PET to assist with the early diagnosis of $\mathrm{AD}$ and to facilitate its use in the monitoring of the efficacy of new treatments for AD. Although validation of the accuracy will need to be separately performed, the proposed MC method can be applied to PET studies of patients with other disorders (e.g., Down syndrome and Parkinson's disease) and to brain PET scans with other molecular imaging probes.

\section{ACKNOWLEDGMENTS}

We thank Dr. Linda Ercoli for performing the neuropsychologic tests, David Truong and Dat $\mathrm{Vu}$ for their computer and database support, the UCLA Cyclotron staff for help with ${ }^{18} \mathrm{~F}$-FDDNP preparation, the UCLA Nuclear Medicine staff for assistance with PET acquisition, and Alex Zhou for his help in the early part of this project. We are also grateful to Dr. John Ashburner at the University College London for his expert technical assistance on the use of SPM2. This work was funded in part by the U.S. Department of Energy contract DE-FG02-02ER63420 and NIH grant P01-AG025831. Dr. Jorge R. Barrio gratefully acknowledges the support of the Elizabeth and Thomas Plott Chair Endowment in Gerontology.

\section{REFERENCES}

1. Buhler P, Just U, Will E, Kotzerke J, van den Hoff J. An accurate method for correction of head movement in PET. IEEE Trans Med Imaging. 2004;23: 1176-1185.

2. Herzog H, Tellmann L, Fulton R, et al. Motion artifact reduction on parametric PET images of neuroreceptor binding. J Nucl Med. 2005;46:1059-1065.

3. Huang S-C, Hoffman EJ, Phelps ME, Kuhl DE. Quantitation in positron emission computed tomography: 2. Effects of inaccurate attenuation correction. J Comput Assist Tomogr. 1979;3:804-814.

4. Logan J, Fowler JS, Volkow ND, Wang GJ, Ding YS, Alexoff DL. Distribution volume ratios without blood sampling from graphical analysis of PET data. J Cereb Blood Flow Metab. 1996;16:834-840.

5. Ruttimann UE, Andreason PJ, Rio D. Head motion during positron emission tomography: is it significant? Psychiatry Res Neuroimaging. 1995;61:43-51. 
6. Small GW, Kepe V, Ercoli LM, et al. PET of brain amyloid and tau in mild cognitive impairment. N Engl J Med. 2006;355:2652-2663.

7. Shin J, Lee SY, Kim SH, Kim YB, Cho SJ. Multitracer PET imaging of amyloid plaques and neurofibrillary tangles in Alzheimer's disease. Neuroimage. 2008; 43:236-244.

8. Folstein MF, Folstein SE, McHugh PR. "Mini-mental state": a practical method for grading the cognitive state of patients for the clinician. J Psychiatr Res. 1975; 12:189-198.

9. McKhann G, Drachman D, Folstein M, Katzman R, Price D, Stadlan EM. Clinical diagnosis of Alzheimer's disease: report of the NINCDS-ADRDA Work Group under the auspices of Department of Health and Human Services Task Force on Alzheimer's Disease. Neurology. 1984;34:939-944.

10. Liu J, Kepe V, Zabjek A, et al. High-yield, automated radiosynthesis of 2-(1$\left\{6-\left[\left(2-\left[{ }^{18} \mathrm{~F}\right]\right.\right.\right.$ fluoroethyl $)($ methyl)amino $\left.]-2-n a p h t h y l\right\}$ ethylidene $)$ malononitrile $\left(\left[{ }^{18} \mathrm{~F}\right] \mathrm{FDDNP}\right)$ ready for animal or human administration. Mol Imaging Biol. 2007;9:6-16.

11. Friston KJ. Statistical Parametric Mapping: The Analysis of Functional Brain Images. 1st ed. Boston, MA: Elsevier/Academic Press; 2007.

12. Abramoff M, Magelhaes $P$, Ram S. Image processing with ImageJ. Biophotonics Int. 2004;11:36-42.

13. Bankman IN. Handbook of Medical Image Processing and Analysis. 2nd ed. Amsterdam, The Netherlands: Elsevier/Academic Press; 2009.
14. Wong K-P, Wardak M, Shao W, et al. Quantitative analysis of $\left[{ }^{18} \mathrm{~F}\right] \mathrm{FDDNP}$ PET using subcortical white matter as reference region. Eur J Nucl Med Mol Imaging. Oct. 31, 2009 [Epub ahead of print]. DOI: 10.1007/s00259-009-1293-8.

15. SPSS, Inc. SPSS Base User's Guide 17.0. Chicago, IL: Prentice Hall; 2007.

16. Van Belle G, Fisher L. Biostatistics: A Methodology for the Health Sciences. 2nd ed. Hoboken, NJ: Wiley-Interscience; 2004.

17. Tellmann L, Fulton R, Bente K, et al. Motion correction of head movements in PET: realisation for routine usage. IEEE Nucl Sci Symp Conf Rec. 2003;5:3105-3107.

18. Andersson JL, Vagnhammar BE, Schneider H. Accurate attenuation correction despite movement during PET imaging. J Nucl Med. 1995;36:670-678.

19. Picard Y, Thompson CJ. Motion correction of PET images using multiple acquisition frames. IEEE Trans Med Imaging. 1997;16:137-144.

20. Costes N, Dagher A, Larcher K, Evans AC, Collins DL, Reilhac A. Motion correction of multi-frame PET data in neuroreceptor mapping: simulation based validation. Neuroimage. 2009;47:1496-1505.

21. Mourik JE, Lubberink M, van Velden FH, Lammertsma AA, Boellaard R. Offline motion correction methods for multi-frame PET data. Eur J Nucl Med Mol Imaging. Jul. 8, 2009 [Epub ahead of print].

22. Bloomfield PM, Spinks TJ, Reed J, et al. The design and implementation of a motion correction scheme for neurological PET. Phys Med Biol. 2003;48:959-978.

23. Mosconi L. Brain glucose metabolism in the early and specific diagnosis of Alzheimer's disease. Eur J Nucl Med Mol Imaging. 2005;32:486-510. 